\title{
Non-genomic actions of estradiol and 4-OH-tamoxifen on murine breast cancer cells
}

\author{
DIEGO RAFFO, OSVALDO PONTIGGIA, ELISA BAL DE KIER JOFFÉ and MARINA SIMIAN
}

Research Area, Institute of Oncology ‘Ángel H. Roffo’, Buenos Aires C1417DTB, Argentina

Received December 3, 2013; Accepted January 7, 2014

DOI: $10.3892 /$ or.2014.3558

\begin{abstract}
Estrogens and tamoxifen do not only exert their effects at the genomic level, but also play a role at the cell membrane activating downstream signaling pathways. We recently characterized an estrogen receptor-positive epithelial murine breast cancer cell line, LM05-E. Utilizing this cell line and MCF-7 cells, we compared the non-genomic effects of estradiol and 4-OH-tamoxifen. We showed that, similar to estradiol, tamoxifen activated the MAPK/ERK 1/2 pathway; however, we did not find activation of PI3K/AKT by either estradiol or tamoxifen. Short-term treatments with estradiol stimulated, whereas tamoxifen inhibited cell proliferation. Using pharmacological inhibitors we showed that the effect of estradiol was mediated by the MAPK/ERK 1/2 pathway, but that inhibition of this pathway did not affect tamoxifen. Surprisingly, however, blocking of PI3K/AKT signaling interfered with the inhibitory effect of tamoxifen. Analysis of the involvement of the EGFR support previous findings that designate this receptor as a mediator of the non-genomic effects of estradiol; blocking EGFR also reverses the inhibitory effect of tamoxifen. Finally, matrix metalloproteinases (MMPs) were confirmed to be involved in the proliferative effect of estradiol. These results demonstrated the novel non-genomic effects of tamoxifen and revealed that pathways downstream of EGFR and PI3K/AKT are involved in the inhibition of cell proliferation. Caution should be exercised when analyzing strategies that aim at combining endocrine therapy with specific signaling inhibitors.
\end{abstract}

\section{Introduction}

Estrogen receptors play a key role in breast cancer progression (1). Seventy-five percent of women diagnosed with breast cancer have estrogen and progesterone receptor (ER,

Correspondence to: Dr Marina Simian, Research Area, Institute of Oncology 'Ángel H. Roffo', San Martín Av. 5481, Buenos Aires C1417DTB, Argentina

E-mail: marina.simian@galuzzi.com

Key words: breast cancer, estradiol, non-genomic signaling, tamoxifen
PR)-positive breast tumors $(2,3)$. Tamoxifen, a selective ER modulator, is the main five-year adjuvant treatment for these patients (4). However, one third of patients treated with tamoxifen will suffer from a recurrence within 15 years (5). Thus, understanding the mechanisms involved in the development of endocrine resistance is of great clinical relevance.

ERs are steroid receptors that are located not only in the nucleus, but also in the cytoplasm, the mitochondria, the endoplasmic reticulum and the plasma membrane (6). Plasma membrane ERs were first identified in endometrial cells (7). In breast cancer cells, the main plasma membrane ER which constitute $\sim 5-10 \%$ of the total ERs, is ER- $\alpha$; little ER- $\beta$ is found at this location (8). Apart from the ERs, several other membrane receptors have been proposed as mediators of the non-genomic actions of estrogens $(6,9,10)$. Nonetheless, ER- $\alpha$ is believed to be the main receptor responsible for rapid signaling in response to estrogens in established experimental conditions in mammary cells (6). ER- $\alpha$ forms a ternary complex together with the IGF-R1 and the adaptor protein SHC in the presence of estradiol (10). Binding of estradiol to ER- $\alpha$ leads to activation of SHC and the formation of the complex followed by the phosphorylation of ER and the IGF-R1 by the c-Src kinase (10). Other authors have proposed a linear pathway implicating activation of the IGF-R1 followed by activation of matrix metalloproteinases (MMPs) which leads to the release of HB-EGF. EGF binds to its receptor leading to activation of the MAPK/ERK $1 / 2$ and PI3K/AKT pathways downstream (11). Notably, few publications to the best of our knowledge, have investigated the effect of tamoxifen on plasma membrane ER and its subsequent non-genomic actions (12).

We developed a mouse model of estrogen-dependent breast cancer from a spontaneous tumor that arose in a female BALB/c mouse in our animal facility (13). This tumor, called M05, is positive for ER and PR and responds to tamoxifen within the first 9 passages (13). From this tumor we established the epithelial cell line LM05-E that is ER- and PR-positive and is responsive to estradiol and tamoxifen as determined by proliferation and apoptosis assays (14). In the present study we investigated the non-genomic effects of estradiol and tamoxifen on the LM05-E cell line. We additionally used MCF-7 cells as a control. We established that, similar to estradiol, tamoxifen activates the MAPK/ERK 1/2 pathway in breast cancer cells; however, we did not find any effect of either estradiol or tamoxifen on the activation status of PI3K/AKT. Short-term treatments with estradiol stimu- 
lated, whereas tamoxifen inhibited, cell proliferation. Using pharmacological inhibitors we showed that, as previously described, the effect of estradiol is mediated by the MAPK/ ERK 1/2 pathway. Inhibition of this pathway did not affect the effect of tamoxifen. Notably, however, blocking of PI3K/AKT signaling interfered with the inhibitory effect of tamoxifen by reversing it. Analysis of the involvement of the EGFR support previous findings that designate this receptor as a mediator of the non-genomic effects of estradiol. Unexpectedly, blocking EGFR also reversed the inhibitory effect of tamoxifen. Finally, MMPs were confirmed as being involved in the proliferative effect of estradiol but not on the inhibitory effect of tamoxifen. These results demonstrated the novel non-genomic effects of tamoxifen and revealed that pathways such as EGFR upstream of PI3K/AKT are involved in inhibition of cell proliferation. Consideration of these mechanisms should be taken into account when analyzing strategies that aim at combining endocrine therapy with specific signaling inhibitors.

\section{Materials and methods}

Cell culture. The MCF-7 and LM05-E cell lines were routinely maintained in growth medium, consisting of DMEM/F12 medium (Sigma-Aldrich), supplemented with $10 \%$ fetal calf serum (FCS; GenSA, Buenos Aires, Argentina) and gentamicin, in a humidified $5 \% \mathrm{CO}_{2} /$ air atmosphere. Serial passages were carried out by treatment of $80 \%$ confluent monolayers with $0.25 \%$ trypsin (Invitrogen) and $0.02 \%$ EDTA in $\mathrm{Ca}^{2+}$-free and $\mathrm{Mg}^{2+}$-free PBS.

Reagents. Estradiol and 4-OH-tamoxifen (both from SigmaAldrich) were prepared 1000X in absolute ethanol and used at a final concentration of $10 \mathrm{nM}$ and $1 \mu \mathrm{M}$, respectively. Controls were subjected to the same dilution of absolute ethanol. The MAPK/ERK 1/2 inhibitor PD98059 (10 $\mu \mathrm{M})$, PI3K/AKT inhibitor LY294002 $(10 \mu \mathrm{M})$, the matrix metalloproteinase inhibitor GM6001 $(10 \mu \mathrm{M})$ and the EGFR inhibitor AG1478 $(6.4 \mu \mathrm{M})$ were all purchased from Calbiochem, and Wortmannin (100 nM) was purchased from Sigma-Aldrich. They were prepared in DMSO, in at least 1000X stock solutions, and the equivalent dilutions of DMSO were used as controls. We previously confirmed the effectiveness of these inhibitors on LM05-E and MCF-7 cells (15).

Cell proliferation studies. Cells (3.0x104/well) were plated in 12 -well plates in growth medium. The next day, cells were washed twice with PBS and the medium was replaced with phenol red-free DMEM/F12. After $48 \mathrm{~h}$, cells were treated with either estradiol $(10 \mathrm{nM})$ or $4-\mathrm{OH}$-tamoxifen $(1 \mu \mathrm{M})$ (or vehicle) for up to $60 \mathrm{~min}$ and were then washed 5 times with PBS as previously published elsewhere (16). Subsequently, cells were cultured for an additional $48 \mathrm{~h}$ in phenol red-free DMEM/F12 supplemented with $1 \%$ charcoal stripped serum. Cells were then counted using a Neubauer chamber. To test the effects of the pharmacological inhibitors on the proliferative response to both estradiol and tamoxifen, starved cells were pretreated with the corresponding inhibitor (or vehicle) for $1 \mathrm{~h}$, and were then subjected to either estradiol or 4-OH-tamoxifen exposure. All experiments were carried out in triplicates and repeated at least three times.
Western immunoblot assay. To evaluate the activation of the different signaling pathways by estradiol and 4-OH-tamoxifen, LM05-E cells $\left(6.0 \times 10^{5}\right)$ were plated on $60-\mathrm{mm}$ plates in growth medium. The next day, cells were washed twice and the medium was replaced by phenol red-free medium. Cells were starved for $48 \mathrm{~h}$ and were then subjected to treatment with estradiol $(10 \mathrm{nM})$ or $4-\mathrm{OH}$-tamoxifen $(1 \mu \mathrm{M})$ for the indicated periods of time. To examine the effect of the specific inhibitors on the activation of the signaling pathways, starved cells were pretreated for $1 \mathrm{~h}$ with the inhibitors (or the corresponding dilution of DMSO) and were subsequently treated with either estradiol or 4-OH-tamoxifen. Protein extracts were prepared by homogenizing cells on ice in RIPA buffer $(50 \mathrm{mM}$ Tris, pH 8.0 containing $150 \mathrm{mM} \mathrm{NaCl}, 0.1 \%$ SDS, $0.5 \%$ deoxycholate and 1\% NP-40) containing protease inhibitors $(40 \mu \mathrm{m}$ phenylmethylsulfonyl fluoride, $5 \mu \mathrm{g} / \mathrm{ml}$ leupeptin, $50 \mu \mathrm{g} / \mathrm{ml}$ aprotinin and $200 \mu \mathrm{M}$ orthovanadate). Protein concentrations were measured using the Bradford method. Samples were mixed with $4 \mathrm{X}$ sample buffer containing $\beta$-mercaptoethanol and boiled for 2 min. Fifty micrograms of each sample was then separated on SDS-PAGE Mini gels (Bio-Rad) and transferred to PVDF membranes (Amersham Biosciences, Uppsala, Sweden). The membranes were blocked overnight in 5\% fatfree milk, $0.1 \%$ Tween-20 in PBS at $4^{\circ} \mathrm{C}$. Primary antibodies were used at a 1/500-1/2,000 dilution in PBS containing 0.1\% Tween-20 (PBST) and 2.5\% fat-free milk, and were incubated at $4^{\circ} \mathrm{C}$ overnight. After washing with PBST, the membranes were incubated with secondary antibodies at a $1 / 1,000$ dilution for $1 \mathrm{~h}$ at room temperature. Signals were detected with an enhanced chemiluminescence kit (ECL; Amersham Biosciences). The following primary antibodies were used: mouse anti-phospho-ERK 1/2, mouse anti-ERK 1/2, rabbit anti-phospho-AKT and rabbit anti-AKT (all from Santa Cruz Biotechnology). The following secondary antibodies were used: donkey-anti rabbit HRP and goat anti-mouse HRP (Santa Cruz Biotechnology). To evaluate the effects of the inhibitors on the activation of the signaling pathways, the inhibitor and vehicle-treated samples were run on the same gel using 15 lane combs.

Statistical analysis. The statistical significance of differences between the groups was calculated by applying one-way ANOVA, followed by Tukey's multiple comparisons test. A value of $\mathrm{P}<0.05$ was considered to indicate a statistically significant result.

\section{Results}

Activation of signaling pathways by estradiol and 4-OH-tamoxifen in LMO5-E cells. To determine the nongenomic responses of LM05-E cells to $17-\beta$-estradiol and 4-OH-tamoxifen, we first examined by western blot analysis the activation of the MAPK/ERK signaling pathway. As previously shown (14), short-term treatments with $10 \mathrm{nM}$ estradiol led to a strong phosphorylation of ERK $1 / 2$ by 10 min that declined to basal levels by $60 \mathrm{~min}$ (Fig. 1A). The same analysis on cells treated with $1 \mu \mathrm{M} 4-\mathrm{OH}$-tamoxifen showed a weaker phosphorylation of ERK at 5-10 min with the signal declining by $60 \mathrm{~min}$ (Fig. 1B). Ethanol-treated cells, in contrast, did not show a significant activation of ERK $1 / 2$ under these experi- 
A

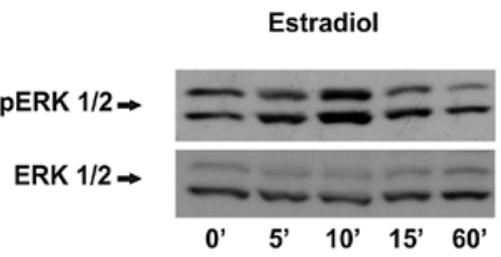

B

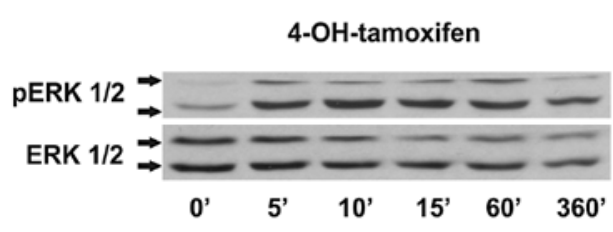

C

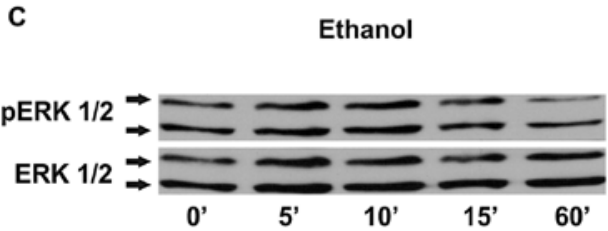

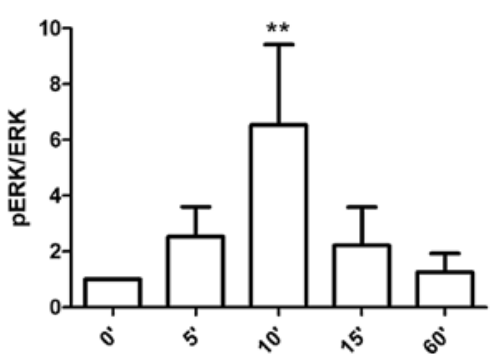
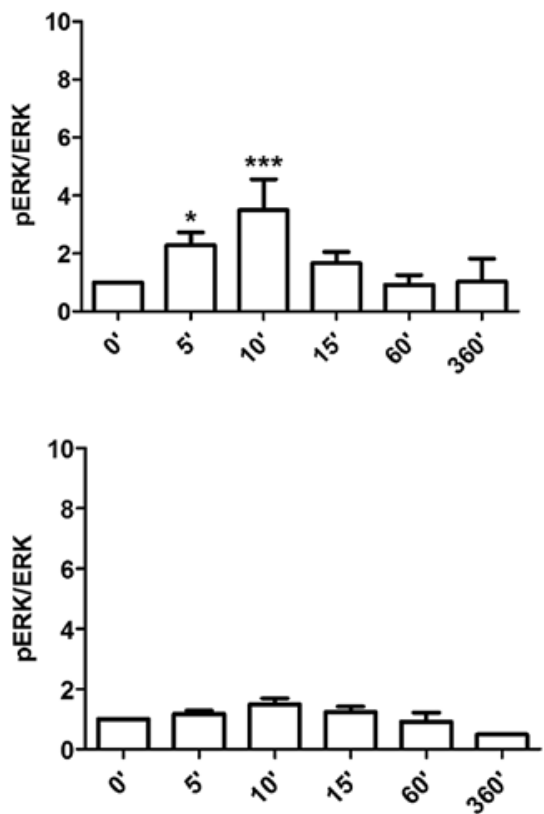

Figure 1. Treatment with estradiol or 4-OH-tamoxifen leads to the rapid phosphorylation of MAPK/ERK 1/2 in LM05-E cells. (A) Time course of ERK $1 / 2$ phosphorylation by estradiol. LM05-E cells plated in $60-\mathrm{mm}$ plates were starved for $48 \mathrm{~h}$ and then treated with $10 \mathrm{nM}$ estradiol for the indicated times. Proteins were extracted with RIPA buffer and analyzed by western blot analysis. A representative western blot analysis is shown on the left together with the densitometric analysis of three independent experiments on the right. A statistically significant activation of ERK was detected at 10 sec $(10 ')\left({ }^{* *} \mathrm{P}<0.01\right)$. (B) Cells starved as in A were treated for the indicated times with $1 \mu \mathrm{M} 4-\mathrm{OH}$-tamoxifen, and the proteins were subsequently analyzed by western blot analysis. The densitometric analysis on the right corresponds to three independent experiments. As with estradiol, a statistically significant activation of ERK $1 / 2$ was detected ( $\mathrm{P}<0.05$ and $\left.{ }^{* * * *} \mathrm{P}<0.001\right)$. (C) Western blot analysis showing lack of activation of ERK $1 / 2$ in the presence of ethanol. To verify that the effects observed for estradiol and tamoxifen were not due to the presence of the vehicle ethanol, or the manipulation of the cells, the protocol was carried out in the presence of ethanol on its own. As shown, no activation of ERK 1/2 was detected under these experimental conditions. Three independent experiments are represented in the graph.

mental conditions (Fig. 1C). To further establish whether the PI3K/AKT pathway is involved, western blots against phosphorylated AKT were carried out on cells receiving the identical treatments. Significant activation of AKT by estradiol or 4-OH-tamoxifen was not detected under our the experimental conditions (Fig. 2A). Moreover, we tested $10 \%$ FCS as a positive control and found that under these conditions no significant AKT activation was observed (Fig. 2B). However, we observed that PI3K inhibitors were able to reduce AKT phosphorylation (Fig. 2C). Thus, our results suggest that estradiol and 4-OH-tamoxifen activate the MAPK/ERK 1/2 pathway in LM05-E cells as previously reported by others in MCF-7 cells (12) and that there was a high basal activation of AKT in our model.

Proliferative response of LM05-E cells to short-term treatments of estradiol and 4-OH-tamoxifen. To investigate the proliferative response of LM05-E cells to short-term treatments of $17-\beta$-estradiol and 4-OH-tamoxifen, we treated cells that had been starved in serum-free medium for $48 \mathrm{~h}$ with $10 \mathrm{nM}$ estradiol, $1 \mu \mathrm{M} 4-\mathrm{OH}$-tamoxifen or vehicle for up to $1 \mathrm{~h}$. The wells were subsequently washed 5 times with PBS, and the medium was replaced by phenol red-free DMEM/F12 supplemented with $1 \%$ charcoal stripped serum as described in Materials and methods and published by others (16). Forty-eight hours later, cells were counted to establish the effect of the treatments on cell proliferation. Treatment with estradiol induced an increase in the total number of cells, and 4-OH-tamoxifen, in contrast, inhibited cell proliferation when compared to the vehicle-treated control (Fig. 3A). Using the same experimental setting, similar results were obtained with MCF-7 cells (Fig. 3B). Given that treatment with 4-OH-tamoxifen reduced cell proliferation between 30 and $50 \%$, we investigated whether significant results could be obtained with shorter periods of treatment. The effect of 4-OH-tamoxifen continued to be significant with treatments as brief as $15 \mathrm{~min}$ (Fig. 3C). To establish whether the effects of the short-term treatments with 4-OH-tamoxifen were being 
A

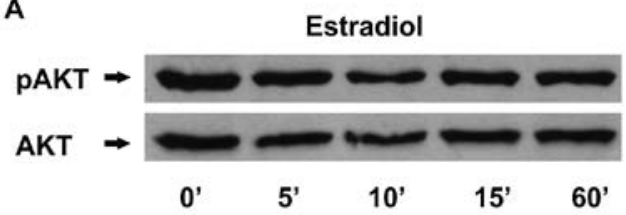

B
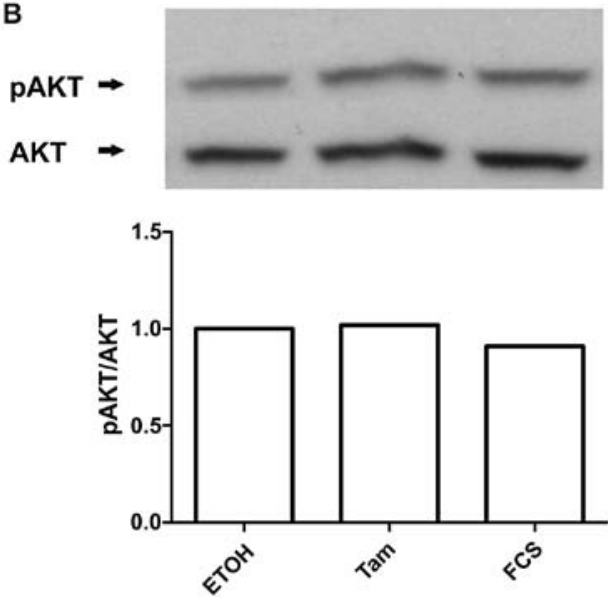
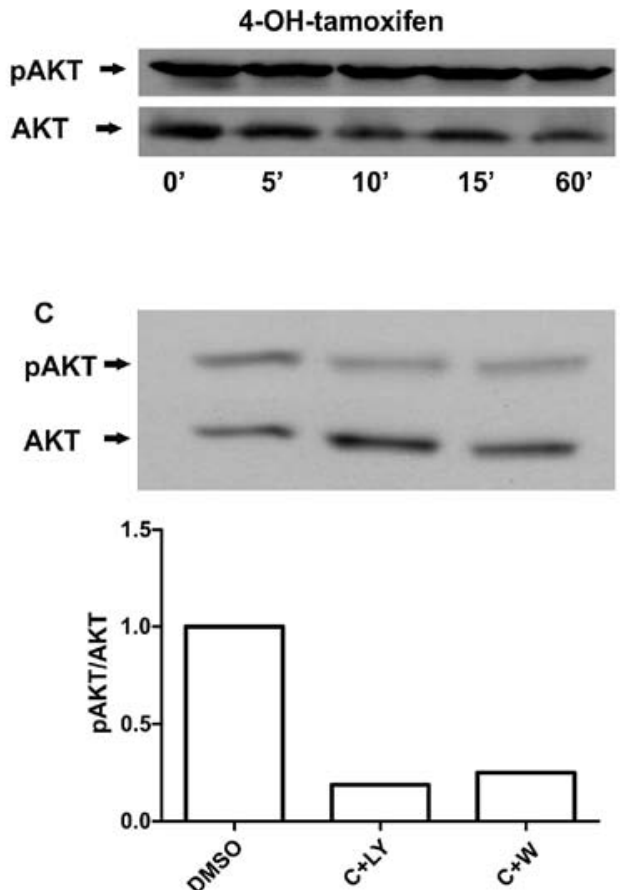

Figure 2. Estradiol and 4-OH-tamoxifen did not activate AKT in LM05-E cells. (A) Time course of AKT phosphorylation by estradiol and 4-OH-tamoxifen. LM05-E cells plated in 60-mm plates were starved for $48 \mathrm{~h}$ and then treated for the indicated times with $10 \mathrm{nM}$ estradiol or $1 \mu \mathrm{M} 4-\mathrm{OH}$-tamoxifen. Proteins were extracted with RIPA buffer and analyzed by western blot analysis. No change in the phosphorylation of AKT was detected by either treatment. One of at least three independent experiments is shown for each treatment. (B) Activation of AKT by tamoxifen or FCS pulses. LM05-E cells plated in 60-mm plates were starved for $48 \mathrm{~h}$ and then treated for 15 min with tamoxifen (Tam) $\left(1.10^{-6} \mathrm{M}\right)$ or $10 \%$ FCS. Proteins were extracted with RIPA buffer and analyzed by western blot analysis. A representative western blot analysis is shown with its corresponding densitometric analysis. (C) Inhibition of AKT by PI3K inhibitors. LM05-E cells plated in 60-mm plates were starved for $48 \mathrm{~h}$ and then treated for $1 \mathrm{~h}$ with medium with LY294002 (LY) (10 $\mu \mathrm{M})$ or Wortmannin (W) (100 nM). Proteins were extracted with RIPA buffer and analyzed by western blot analysis. A representative western blot analysis with its densitometric analysis is shown.

mediated through the same receptor as estradiol, LM05-E cells were pre-treated for $15 \mathrm{~min}$ with $10 \mathrm{nM}$ estradiol and subsequently $1 \mu \mathrm{M} 4-\mathrm{OH}$-tamoxifen was added for $1 \mathrm{~h}$. Fortyeight hours later, the effect on cell proliferation showed that estradiol only partially counteracted the effects of tamoxifen (Fig. 3D), suggesting that the ER is involved in this mechanism. However, it has also been proposed by other authors, that tamoxifen may exert its effects through ER-dependent and -independent mechanisms (17).

The MAPK/ERK pathway is involved in the proliferative response to estradiol whereas the PI3K/AKT pathway mediates the inhibitory effect of 4-OH-tamoxifen. We established above that short-term treatments with estradiol stimulated while 4-OH-tamoxifen inhibited the proliferation of LM05-E cells; however, both effects were accompanied by an increase in ERK phosphorylation and no changes in AKT phosphorylation. To further establish the involvement of the downstream signaling pathways, we pre-treated LM05-E cells with the MEK inhibitor PD98059 $(10 \mu \mathrm{M})$ or the PI3K inhibitors LY294002 $(10 \mu \mathrm{M})$ and Wortmannin $(100 \mathrm{nM})$ and then subjected them to short pulses of estradiol and 4-OH-tamoxifen as explained above. Pre-treatment with PD98059 abrogated the stimulatory effects of estradiol on cell proliferation (Fig. 4A), but did not affect the inhibitory effect of 4-OH-tamoxifen (Fig. 4B). On the other hand, LY294002 did not affect the stimulatory effect of estradiol (Fig. 4C). However, inhibition of the PI3K/AKT pathway with either LY294002 or Wortmannin reversed the inhibitory effect of 4-OH-tamoxifen (Figs. 4D and 5A). Pretreatment with Wortmaninn or LY294002 also reversed the inhibitory effect of 4-OH-tamoxifen on MCF-7 cells (Fig. 5B and C); however, the MEK inhibitor, as in the case of LM05-E cells, did not have an impact on the effect of the anti-estrogen (Fig. 5D). Our results thus suggest that the MAPK/ERK pathway plays a key role in mediating the stimulatory effect of estradiol whereas the PI3K/AKT pathway is involved in the inhibitory effect of 4-OH-tamoxifen.

EGFR mediates the downstream effects of both estradiol and 4-OH-tamoxifen, while MMPs are only involved in the effects of estradiol. It has been previously shown in MCF-7 cells that binding of estradiol to membrane ER leads to the shedding of HB-EGF by MMPs and the subsequent activation of the MAPK/ERK pathway (11). To investigate whether EGFR is involved in the proliferative response to short-term treatment with estradiol or 4-OH-tamoxifen in LM05-E cells, we preincubated the cells with the EGFR inhibitor AG1478 $(6.4 \mu \mathrm{M})$ and then incubated the cells with estradiol or 4-OH-tamoxifen for $1 \mathrm{~h}$. The EGFR inhibitor completely blocked the increase in cell number induced by estradiol (Fig. 6A). Western blot analysis showed that inhibition of EGFR led to reduced activation of MAPK/ERK (Fig. 6B). Moreover, it abrogated the inhibitory effect of 4-OH-tamoxifen in both LM05-E (Fig. 6C) and MCF-7 cells (data not shown) as previously revealed (12). Western blot analysis showed that 4-OH-tamoxifen induced MAPK/ERK 1/2 activation was also inhibited (Fig. 6D) 

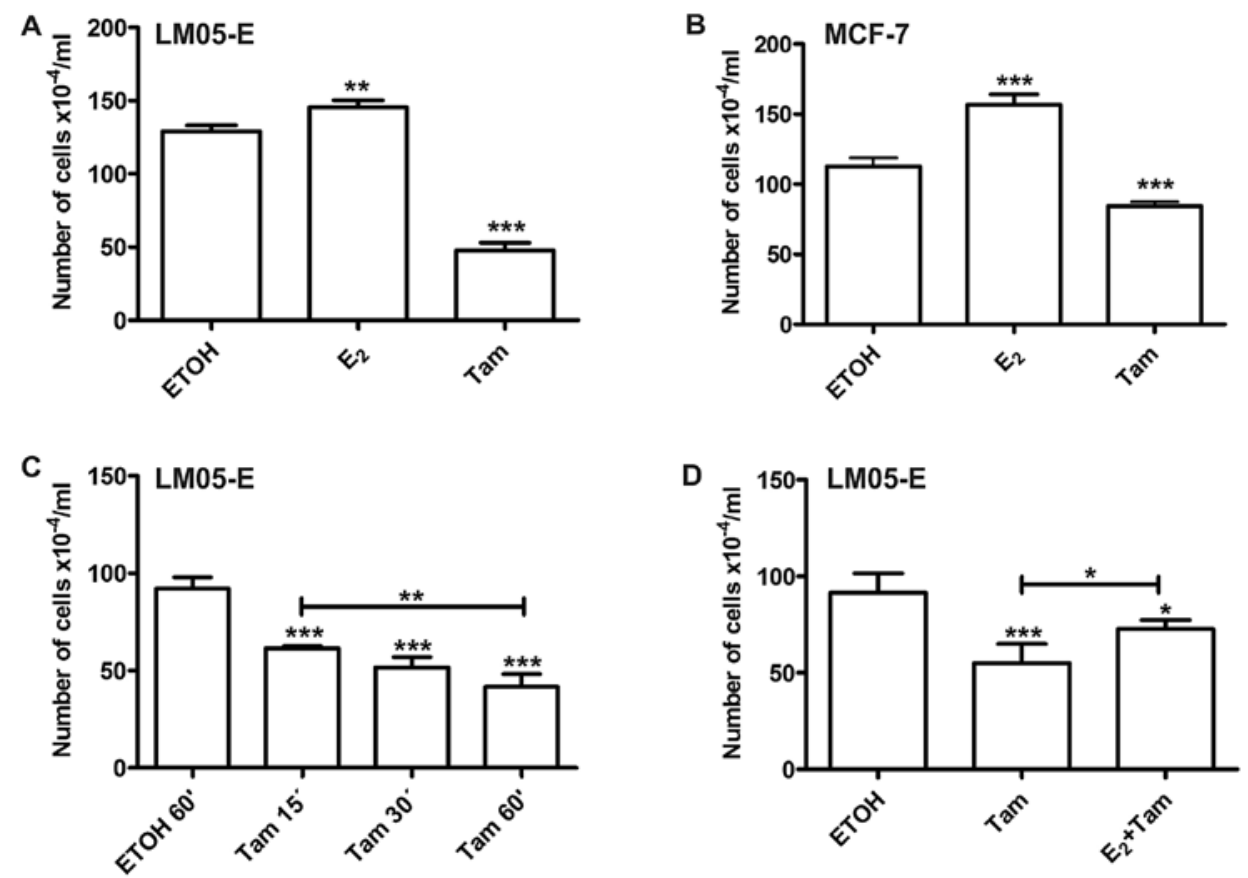

Figure 3. Short-term treatments with estradiol and 4-OH-tamoxifen modulate the proliferation of LM05-E cells. Cells starved for $48 \mathrm{~h}$ in 12 -well plates were treated for up to $1 \mathrm{~h}$ with $10 \mathrm{nM}$ estradiol or $1 \mu \mathrm{M} 4-\mathrm{OH}$-tamoxifen in culture media in the absence of serum, washed, and counted $48 \mathrm{~h}$ later as described in Materials and methods. (A) A statistically significant increase in cell number was detected in the LM05-E cells treated with estradiol $\left(\mathrm{E}_{2}\right)$. 4-OH-tamoxifen (Tam) had an inhibitory effect as expected. Controls were treated with the vehicle ethanol $(\mathrm{ETOH})\left({ }^{* *} \mathrm{P}<0.01 ;{ }^{* * * *} \mathrm{P}<0.001\right)$. (B) The same experimental setting was used as in A on MCF-7 cells. Similar results were obtained with an increase in cell number in the presence of $\mathrm{E}_{2}$ and a reduction following treatment with 4-OH-tamoxifen $\left({ }^{* * *} \mathrm{P}<0.001\right)$. (C) 4-OH-tamoxifen led to a statistically significant inhibition of cell proliferation with treatments as short as 15 min $(15 ')$. The same experimental setting was used as above, with reduced exposure time to 4-OH-tamoxifen $\left({ }^{* *} \mathrm{P}<0.01 ;{ }^{* * * *} \mathrm{P}<0.001\right)$. (D) Treatment with estradiol $\left(\mathrm{E}_{2}\right)$ partially counteracted the effect of 4-OH-tamoxifen (Tam). LM05-E cells were starved and pre-incubated with estradiol for 15 min before being exposed to 4-OH-tamoxifen for $1 \mathrm{~h}$ as explained above. A statistically significant partial reversal of the inhibitory effect of 4-OH-tamoxifen was achieved ("P $<0.05$; $\left.{ }^{* * * *} \mathrm{P}<0.001\right)$ suggesting that this SERM exerts its effects through ER-dependent and -independent mechanisms. One of three representative experiments is shown for all experiments.

A

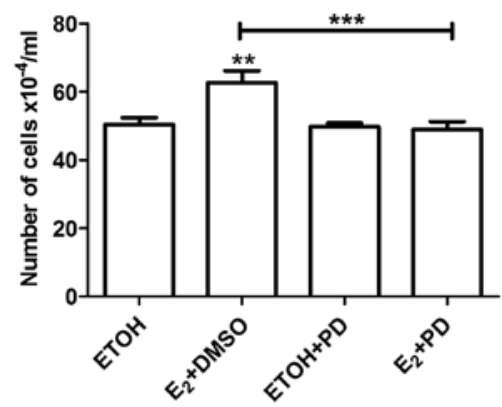

C

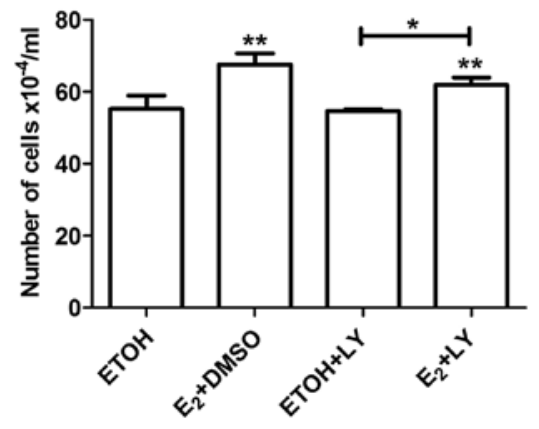

B

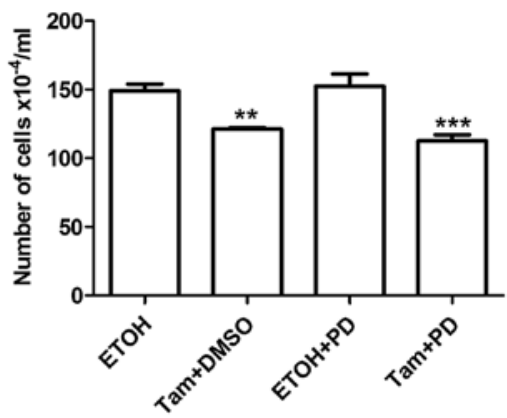

D

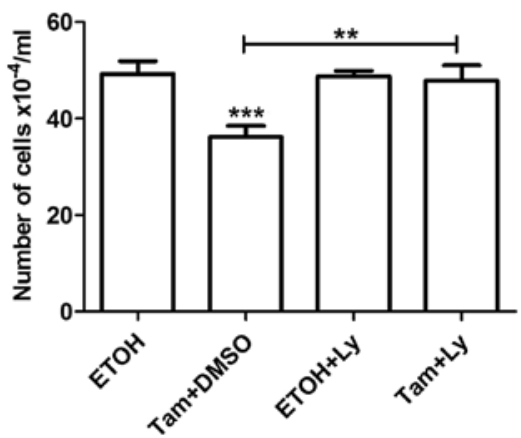

Figure 4. Role of the MAPK/ERK and PI3K/AKT pathways in the non-genomic effects of estradiol on LM05-E cells. (A) LM05-E cells starved for $48 \mathrm{~h}$ were pre-incubated with the MAPK/ERK inhibitor PD98059 (PD) (10 $\mu \mathrm{M})$ or vehicle (DMSO) for $1 \mathrm{~h}$ before being exposed to short-term treatment with estradiol $\left(\mathrm{E}_{2}\right)$. Cells were then washed and incubated as described in Materials and methods and counted $48 \mathrm{~h}$ later. PD inhibited the increase in cell number induced by estradiol in a statistically significant manner $\left.{ }^{(* * *} \mathrm{P}<0.001\right)$. It had no effect on its own. (B) Starved LM05-E cells were pre-incubated with PD as explained above and subsequently treated with 4-OH-tamoxifen (Tam) for $1 \mathrm{~h}$ and counted $48 \mathrm{~h}$ later. PD had no influence on the inhibitory effect of tamoxifen $\left({ }^{* *} \mathrm{P}<0.01\right.$; $\left.{ }^{* * * *} \mathrm{P}<0.001\right)$. (C) LM05-E cells were starved and pre-treated with the PI3K/AKT inhibitor LY294002 (LY) (10 $\left.\mu \mathrm{M}\right)$. LY did not a have statistically significant effect on the proliferation induced by estradiol $\left({ }^{*} \mathrm{P}<0.05 ;{ }^{* *} \mathrm{P}<0.01\right)$. (D) However, pre-treatment with LY reversed the inhibitory effect of 4-OH-tamoxifen on LM05-E cells $\left({ }^{* *} \mathrm{P}<0.01 ;{ }^{* * *} \mathrm{P}<0.001\right)$. 

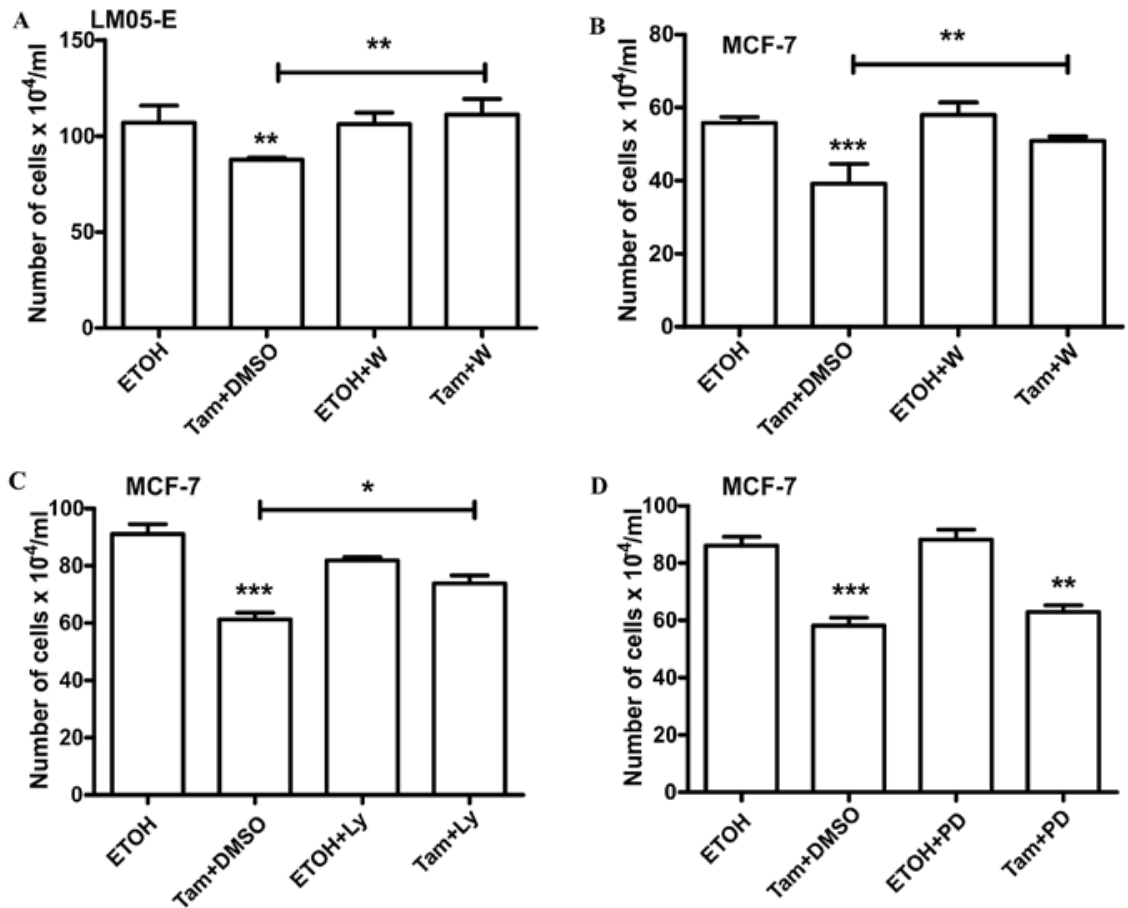

Figure 5. Role of the MAPK/ERK and PI3K/AKT pathways in the non-genomic effects of 4-OH-tamoxifen and estradiol on LM05-E and MCF-7 cells (A) LM05-E cells starved for $48 \mathrm{~h}$ were pre-incubated with the PI3K inhibitor Wortmannin (W) (100 nM) or vehicle (DMSO) for $1 \mathrm{~h}$ before being exposed to short-term treatments with 4-OH-tamoxifen (Tam). They were then washed and incubated as described in Materials and methods and counted $48 \mathrm{~h}$ later. Wortmannin reversed the inhibitory effect of $4-\mathrm{OH}$-tamoxifen $\left({ }^{* *} \mathrm{P}<0.01\right)$. It had no effect on its own. (B) Starved MCF-7 cells were pre-incubated with Wortmannin as explained above and subsequently treated with 4-OH-tamoxifen for $1 \mathrm{~h}$ and counted $48 \mathrm{~h}$ later. Wortmannin reversed the inhibitory effect of 4-OH-tamoxifen $\left({ }^{* *} \mathrm{P}<0.01 ;{ }^{* * *} \mathrm{P}<0.001\right)$. It had no effect on its own. (C) MCF-7 cells were starved and pre-treated with the PI3K/AKT inhibitor LY294002 (LY) $(10 \mu \mathrm{M})$; pre-treatment with LY reversed the inhibitory effect of 4-OH-tamoxifen on MCF-7 cells ( $\left.{ }^{*} \mathrm{P}<0.05 ;{ }^{* * * *} \mathrm{P}<0.001\right)$. (D) Pre-treatment of MCF-7 cells with the MAPK/ERK inhibitor PD98059 (PD) $(10 \mu \mathrm{M})$ did not disturb the inhibitory effect of 4-OH-tamoxifen $\left({ }^{* *} \mathrm{P}<0.01 ;{ }^{* * * *} \mathrm{P}<0.001\right)$. One of three experiments is shown in each case.
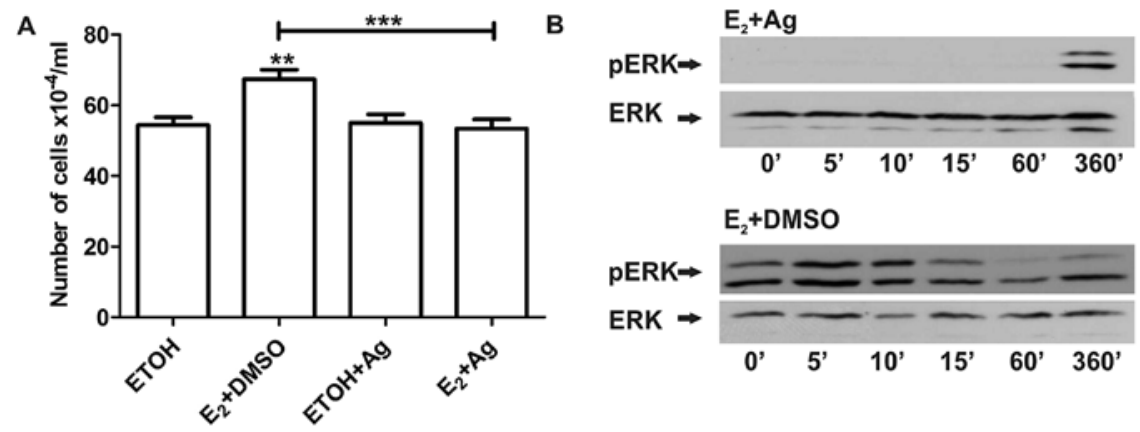

C

D
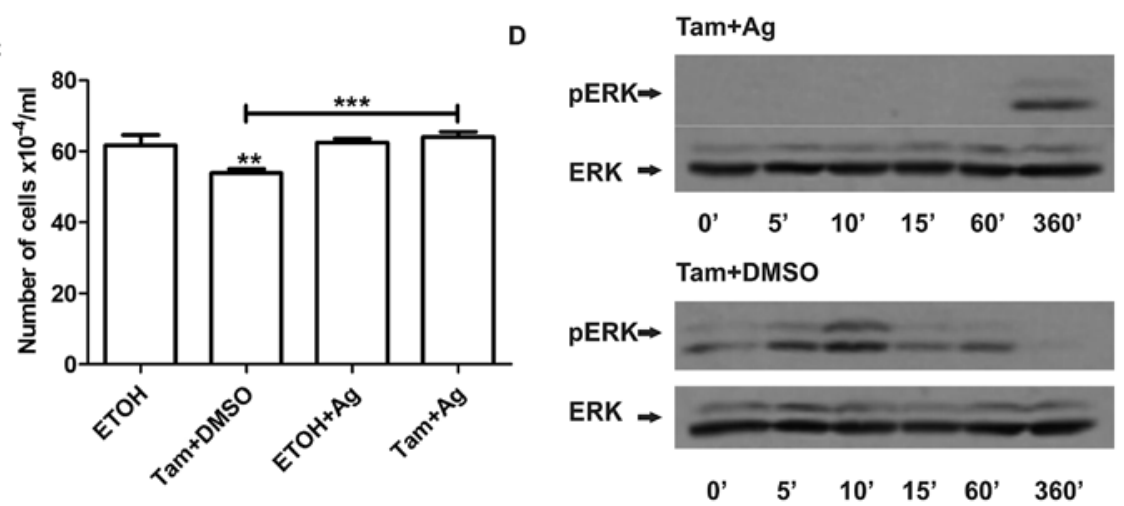

Figure 6. Involvement of the EGFR pathway in the non-genomic effects of estradiol and 4-OH-tamoxifen. LM05-E cells were starved and pre-treated for $1 \mathrm{~h}$ with the EGFR inhibitor AG1478 (AG) $(6.4 \mu \mathrm{M})$. Subsequently, they were either treated with estradiol $\left(\mathrm{E}_{2}\right)$ or 4-OH-tamoxifen (Tam) and analyzed for cell proliferation $48 \mathrm{~h}$ later, or MAPK/ERK phosphorylation within $6 \mathrm{~h}$ of treatment. (A) AG had a statistically significant inhibitory effect on cell proliferation induced by estradiol $\left(\mathrm{E}_{2}\right)\left({ }^{* *} \mathrm{P}<0.01 ;{ }^{* * *} \mathrm{P}<0.001\right)$. (B) This effect was associated with strong inhibition of ERK $1 / 2$ phosphorylation as shown by western blot analysis. As in A, the control cells were pre-treated with DMSO. (C) As in the case of estradiol, AG counteracted the effect of 4-OH-tamoxifen. A statistically significant reversal of the inhibitory effect of $4-\mathrm{OH}$-tamoxifen on cell proliferation was detected $\left({ }^{* *} \mathrm{P}<0.01 ;{ }^{* * * *} \mathrm{P}<0.001\right)$. (D) Western blot analysis showed that phosphorylation of ERK induced by Tam was inhibited by AG, compared to the vehicle control treated with DMSO. One of three experiments is shown in each case. 

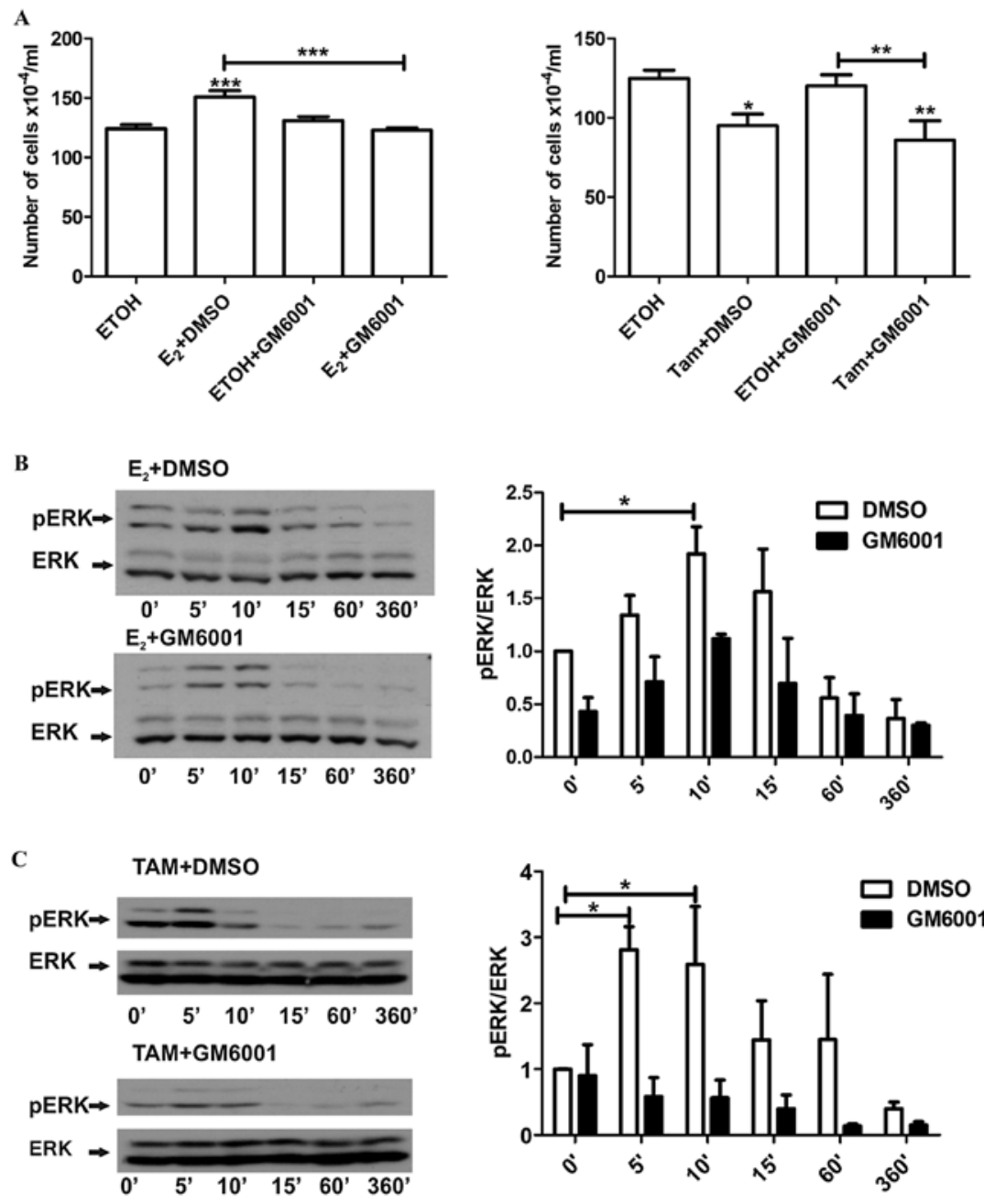

Figure 7. MMPs are mediators of the non-genomic effects of estradiol but not of 4-OH-tamoxifen. LM05-E cells were starved for $48 \mathrm{~h}$ and pre-treated for $1 \mathrm{~h}$ with the MMP inhibitor GM6001 (10 $\mu \mathrm{M})$. Subsequently, they were either treated with estradiol $\left(\mathrm{E}_{2}\right)$ or 4-OH-tamoxifen (Tam) and analyzed for cell proliferation $48 \mathrm{~h}$ later or for MAPK/ERK phosphorylation within $6 \mathrm{~h}$ of treatment. (A) GM6001 showed a statistically significant inhibitory effect on cell proliferation induced by the non-genomic effects triggered by estradiol $\left({ }^{* *} \mathrm{P}<0.01 ;{ }^{* * *} \mathrm{P}<0.001\right)$, but did not affect the inhibitory effect of 4-OH-tamoxifen (right). (B) Western blot analysis revealed a delay in ERK 1/2 phosphorylation upon pre-incubation with GM6001 and subsequent estradiol treatment. The control was treated with the vehicle DMSO. The graph on the right represents the results of three independent experiments ( $\mathrm{P}<0.05)$. (C) Pre-treatment with GM6001 also delayed the activation of MAPK/ERK by 4-OH tamoxifen even though it did not affect the inhibition of proliferation $\left({ }^{*} \mathrm{P}<0.05\right)$. The mean of three experiments is shown in the graph on the right.

suggesting that EGFR provides a point of convergence for both the stimulatory and inhibitory effects of these compounds, even though MAPK/ERK 1/2 appears to play a key role only in estradiol-induced cell proliferation.

To determine whether shedding of HB-EGF is also a common event leading to these distinct effects, LM05-E cells were pre-incubated with the MMP inhibitor GM6001 $(10 \mu \mathrm{M})$ and then treated with either estradiol or 4-OH-tamoxifen. GM6001, as did AG1478, led to a reversal of the stimulatory effect of estradiol, but did not affect the inhibitory effect of 4-OH-tamoxifen (Fig. 7A). Moreover, as noted in the western blot analysis, the MMP inhibitor consistently delayed the phosphorylation of ERK in the cells treated with estradiol (Fig. 7B); notably, this was also the case for the cells treated with 4-OH-tamoxifen (Fig. 7C). Thus, our results showed that similar to MCF-7 cells, the non-genomic effects of estradiol involve MMP activity and the participation of EGFR. Moreover, our results suggest that $4-\mathrm{OH}$-tamoxifen also requires an active EGFR to exert its biological effects even though MMP activity does not appear to play a role.

\section{Discussion}

In the present study, we investigated the non-genomic responses of murine LM05-E breast cancer cells to estradiol and 4-OH-tamoxifen. Through proliferation assays, based on a previously published method established by Vallejo et al (16) and western blot analysis, we determined that, as previously shown for MCF-7 and T47D cells, shortterm exposure to estradiol and 4-OH-tamoxifen led to the phosphorylation of MAPK/ERK 1/2. As expected, treatment with estradiol had a positive impact on cell number at $48 \mathrm{~h}$, whereas 4-OH-tamoxifen had an inhibitory effect. Estradiol partially counteracted the inhibitory effect of 4-OH-tamoxifen suggesting that the ER is at least partially involved in tamoxifen membrane-initiated signaling. Analysis 
of the active involvement of the signaling pathways on the non-genomic responses using specific inhibitors revealed that MAPK/ERK 1/2 mediates the proliferative response to estradiol, but not the inhibitory effect of 4-OH-tamoxifen. On the other hand, we did not find an involvement of PI3K/AKT in the response to estradiol; surprisingly, however, LY294002 reversed the inhibitory effect of 4-OH-tamoxifen, suggesting that PI3K/AKT mediates the inhibitory effect of tamoxifen. Moreover, the PI3K inhibitor Wortmannin also abolished the inhibitory effect of tamoxifen, indicating that it is most probably due to PI3K inhibition and not other off-targets of LY294002, such as CK2 or GSK3 $\beta$ (18). Blocking EGFR strongly inhibited the activation of MAPK/ERK 1/2 induced by both estradiol and 4-OH-tamoxifen. With regards to proliferation, EGFR mediated the stimulatory effect of estradiol, as previously shown by others, and, as in the case of the PI3K/ AKT pathway, its constraint neutralized the inhibitory effect of 4-OH-tamoxifen. Finally, we found that the MMP inhibitor GM6001 delayed the activation of ERK 1/2 by estradiol and 4-OH-tamoxifen but had an impact only on the proliferative effect of estradiol.

Previously, several studies demonstrated that short-term treatment with estradiol led to the activation of the MAPK/ERK $1 / 2$ pathway in human breast cancer cells and other cell types such as endothelial cells $(11,19)$. Few authors have investigated the non-genomic effects of 4-OH-tamoxifen and reported rapid activation of MAPK/ERK $1 / 2$ by this SERM (12). Using LM05-E cells, we found that similar results were obtained regarding the MAPK/ERK 1/2 pathway, with rapid MMP-dependent phosphorylation following both estradiol and 4-OH-tamoxifen treatments. We did not detect, however, any changes in AKT phosphorylation for either treatment or FCS pulses. This may be due to a high basal activation of AKT in our model. Nevertheless, activation of AKT by estradiol has been described for MCF-7 cells by Razandi et al (11). Together with the activation of the signaling pathways, we investigated the impact of each of these on cell proliferation as a measure of the long-term impact on cell behavior. As expected, $48 \mathrm{~h}$ after exposure to estradiol, a statistically significant increase in cell number was detected; conversely a decrease was found in culture plates treated with 4-OH-tamoxifen. Blocking the MAPK/ERK 1/2 pathway had a direct impact on the increase in cell number induced by estradiol, however, it did not affect the inhibitory effect of 4-OH-tamoxifen. This is contrary to the findings reported by Zheng et al (12) who described a reduction in cell death induced by $4-\mathrm{OH}$-tamoxifen in cells pre-treated with PD98059. However, the concentrations of both 4-OH-tamoxifen and the MAPK/ERK inhibitors used by the other authors were higher than those used in the present study ( 5 and $20 \mu \mathrm{M}$, respectively), and the number of dead cells were counted up to $60 \mathrm{~min}$ after the initiation of the treatments as their end point. In contrast, we evaluated the effect of the treatments on cell number $48 \mathrm{~h}$ later. Most interesting, however, was the finding that blocking PI3K/AKT counteracted the effect of 4-OH-tamoxifen. As mentioned above, we did not find a statistically significant change in AKT phosphorylation following treatment with either estradiol or 4-OH-tamoxifen on LM05-E cells, and LY294002 did not affect the increase in cell number induced by estradiol. In addition, we found that both LY294002 and Wortmannin reduced basal AKT activity.
Other studies found increased phosphorylation of AKT as a result of non-genomic effects of estradiol $(11,19)$. Notably, $\mathrm{PI} 3 \mathrm{~K} / \mathrm{AKT}$ inhibition has been shown to promote resistance to chemotherapeutic drugs in MDA-MB-231 breast cancer cells as well as in other cell types such as DU-145 prostate, HCT-116 colon and A-549 lung carcinoma cells (20). The authors found that this effect was only present when the cells were pre-incubated with the PI3K/AKT inhibitor. On the contrary, when the timing was changed, with cells being exposed to LY294002 after treatment with chemotherapeutic drugs, cell survival was decreased. Based on the same line of evidence, we found that inhibition of EGFR also reversed the inhibitory effect of 4-OH-tamoxifen but additionally had an impact on the proliferative effect of estradiol. Western blot analysis showed that AG1478 completely blocked the MAPK/ERK 1/2 pathway and this could account for the interference of the proliferative effect of estradiol. However, we did not find any change in the status of the PI3K/AKT pathway (data not shown) in the presence of AG1478 suggesting that some other pathway downstream of EGFR was accountable for the observed effect. Other authors have recently shown that inhibition of EGFR by various inhibitors increases ErbB3 protein levels in breast cancer cells and downregulates FAS which is involved in apoptotic cell death (21). On the other hand, HER2/HER3 heterodimer formation has been shown to be involved in EGFR inhibitor resistance in lung cancer cells (22).

Analysis of the effect of the MMP inhibitor GM6001 on cells treated with estradiol and 4-OH-tamoxifen showed that only in the case of estradiol did GM6001 have an effect. However, western blot analysis revealed that the MMP inhibitor delayed the phosphorylation of MAPK/ERK $1 / 2$ induced by both estradiol and 4-OH-tamoxifen. The fact that at the proliferative level only cells treated with estradiol were affected supports our previous conclusion of the involvement of the MAPK/ERK 1/2 pathway as a mediator of the effect of estradiol, but not of tamoxifen.

Our results demonstrated that the selective estrogen receptor modulator 4-OH-tamoxifen stimulates non-genomic signaling in murine breast cancer cells. Even though the MAPK/ERK 1/2 pathway is activated as with estradiol, it does not appear to be a mediator of the effect of tamoxifen. Moreover, the PI3K/AKT pathway does not appear to be altered by tamoxifen treatment; however, its inhibition reverses the inhibitory effect of tamoxifen as does the blockage of the EGFR. These results suggest that caution should be exercised when considering the possibility of combining anti-estrogens with signaling pathway inhibitors as the latter may interfere with the non-genomic effects of tamoxifen and counteract part of its inhibitory effect.

\section{Acknowledgements}

The present study is supported by grants from the Susan G. Komen Foundation (BCTR0600341), Préstamo BID PICT 2008-0325 and the Florencio Fiorini Foundation to M.S., and grants UBACYT 2011-2014 (M0243) and Préstamo BID PICT 2010-01296 to E.B.K.J.

\section{References}

1. Russo J and Russo IH: The role of estrogen in the initiation of breast cancer. J Steroid Biochem Mol Biol 102: 89-96, 2006. 
2. Harvey JM, Clark GM, Osborne CK and Allred DC: Estrogen receptor status by immunohistochemistry is superior to the ligandbinding assay for predicting response to adjuvant endocrine therapy in breast cancer. J Clin Oncol 17: 1474-1481, 1999.

3. Graham JD, Yeates C, Balleine RL, et al: Characterization of progesterone receptor $\mathrm{A}$ and $\mathrm{B}$ expression in human breast cancer. Cancer Res 55: 5063-5068, 1995.

4. Sengupta S and Jordan VC: Selective estrogen modulators as an anticancer tool: mechanisms of efficiency and resistance. Adv Exp Med Biol 630: 206-219, 2008.

5. Musgrove EA and Sutherland RL: Biological determinants of endocrine resistance in breast cancer. Nat Rev Cancer 9: 631-643, 2009.

6. Levin ER and Pietras RJ: Estrogen receptors outside the nucleus in breast cancer. Breast Cancer Res Treat 108: 351-361, 2008.

7. Pietras RJ and Szego CM: Specific binding sites for oestrogen at the outer surfaces of isolated endometrial cells. Nature 265 69-72, 1977.

8. Pedram A, Razandi M and Levin ER: Nature of functional estrogen receptors at the plasma membrane. Mol Endocrinol 20: 1996-2009, 2006.

9. Toran-Allerand CD, Guan X, MacLusky NJ, et al: ER-X: a novel, plasma membrane-associated, putative estrogen receptor that is regulated during development and after ischemic brain injury. J Neurosci 22: 8391-8401, 2002.

10. Song RX, Fan P, Yue W, Chen Y and Santen RJ: Role of receptor complexes in the extranuclear actions of estrogen receptor $\alpha$ in breast cancer. Endocr Relat Cancer 13 (Suppl 1): S3-S13, 2006.

11. Razandi M, Pedram A, Park ST and Levin ER: Proximal events in signaling by plasma membrane estrogen receptors. J Biol Chem 278: 2701-2712, 2003.

12. Zheng A, Kallio A and Harkonen P: Tamoxifen-induced rapid death of MCF-7 breast cancer cells is mediated via extracellularly signal-regulated kinase signaling and can be abrogated by estrogen. Endocrinology 148: 2764-2777, 2007.

13. Simian M, Manzur T, Rodriguez V, de Kier Joffe EB and Klein S: A spontaneous estrogen-dependent, tamoxifen-sensitive mouse mammary tumor: a new model system to study hormoneresponsiveness in immune competent mice. Breast Cancer Res Treat 113: 1-8, 2009
14. Pontiggia O, Rodriguez V, Fabris V, et al: Establishment of an in vitro estrogen-dependent mouse mammary tumor model: a new tool to understand estrogen responsiveness and development of tamoxifen resistance in the context of stromal-epithelial interactions. Breast Cancer Res Treat 116: 247-255, 2009.

15. Pontiggia O, Sampayo R, Raffo D, et al: The tumor microenvironment modulates tamoxifen resistance in breast cancer: a role for soluble stromal factors and fibronectin through $\beta 1$ integrin. Breast Cancer Res Treat 133: 459-471, 2012.

16. Vallejo G, Ballare C, Baranao JL, Beato $M$ and Saragueta P: Progestin activation of nongenomic pathways via cross talk of progesterone receptor with estrogen receptor $\beta$ induces proliferation of endometrial stromal cells. Mol Endocrinol 19: 3023-3037, 2005.

17. Ferlini C, Scambia G, Marone M, et al: Tamoxifen induces oxidative stress and apoptosis in oestrogen receptor-negative human cancer cell lines. Br J Cancer 79: 257-263, 1999.

18. Gharbi SI, Zvelebil MJ, Shuttleworth SJ, et al: Exploring the specificity of the PI3K family inhibitor LY294002. Biochem J 404: 15-21, 2007

19. Stice JP, Mbai FN, Chen L and Knowlton AA: Rapid activation of nuclear factor $\kappa \mathrm{B}$ by $17 \beta$-estradiol and selective estrogen receptor modulators: pathways mediating cellular protection. Shock 38: 128-136, 2012.

20. McDonald GT, Sullivan R, Pare GC and Graham CH: Inhibition of phosphatidylinositol 3-kinase promotes tumor cell resistance to chemotherapeutic agents via a mechanism involving delay in cell cycle progression. Exp Cell Res 316: 3197-3206, 2010.

21. Grovdal LM, Kim J, Holst MR, Knudsen SL, Grandal MV and van Deurs B: EGF receptor inhibitors increase ErbB3 mRNA and protein levels in breast cancer cells. Cell Signal 24: 296-301, 2012.

22. Hirata A, Hosoi F, Miyagawa M, et al: HER2 overexpression increases sensitivity to gefitinib, an epidermal growth factor receptor tyrosine kinase inhibitor, through inhibition of HER2/ HER3 heterodimer formation in lung cancer cells. Cancer Res 65: 4253-4260, 2005. 